\title{
Rescuing memory
}

A recent paper published in PNAS has shown that upregulation of brainderived neurotrophic factor (BDNF), using a compound that modulates glutamate receptors, improves memory impairments in a mouse model of Huntington's disease (HD).

$\mathrm{HD}$ is caused by a CAG repeat expansion in HTT, the gene that encodes the huntingtin protein, and is characterized by severe motor defects, as well as cognitive and memory deficits. Previous studies have indicated that actin polymerization in dendritic spines, which normally stabilizes long-term potentiation (LTP), is defective in a genetic knockin mouse model of HD. BDNF is known to facilitate LTP by promoting activity-driven actin polymerization in dendritic spines and, given that reduced levels of BDNF are found in this mouse model and in patients with $\mathrm{HD}$, the authors investigated whether impaired BDNF signalling in HD could disrupt the synapses that are needed to encode memory.

To examine this, they used CX929, an allosteric modulator of a-amino-3-hydroxy-5-methyl4-isoxazole propionic acid (AMPA)-type glutamate receptors (also known as an ampakine) that increases excitatory transmission and thereby upregulates BDNF expression. When CX929 was injected twice daily for 4 days, HD knock-in mice (CAG140) displayed upregulated hippocampal BDNF levels and robust LTP in hippocampal slices.

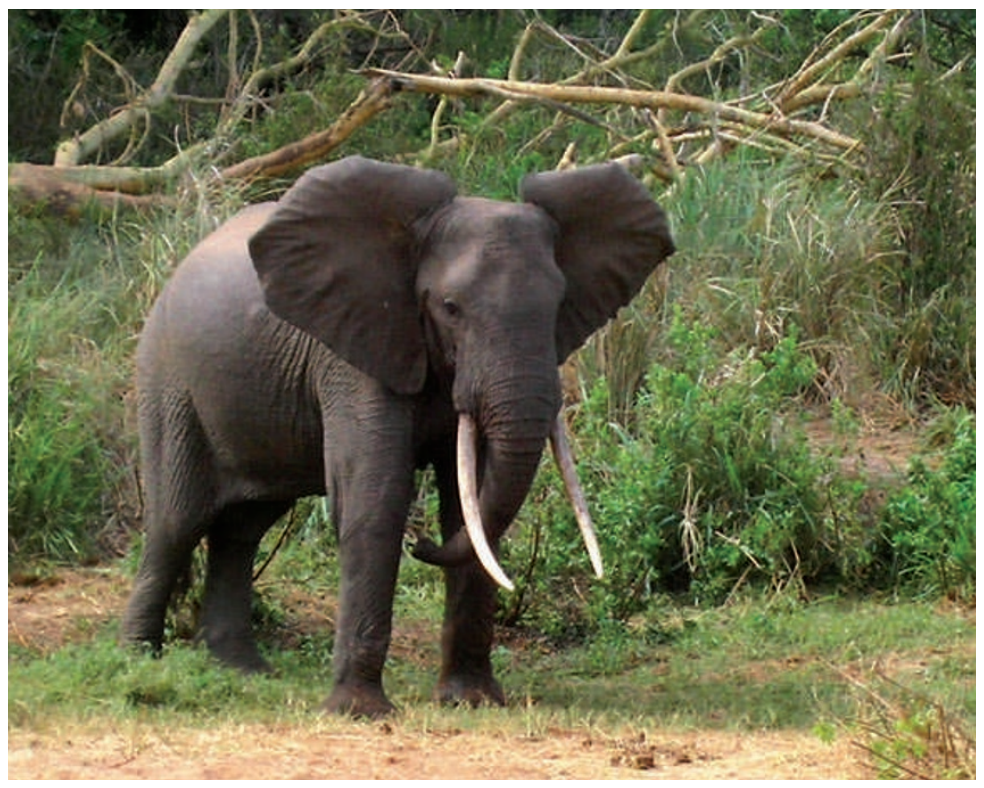

Next, the authors tested whether CX929 could reverse the impaired actin polymerization in dendritic spines of the CAG140 mice. Whereas hippocampal slices prepared from vehicle-treated mice showed a low level of labelled dendritic spines in response to neuronal stimulation, CX929 treatment increased the number of densely labelled spines, indicating that CX929 restored activity-driven actin polymerization.

Finally, the authors examined the effects of CX929 treatment on the behaviour of CAG140 mice. In learning tasks, treated mice showed experience-dependent changes in behaviour, and in novelobject recognition tests of long-term memory, CX929 reduced long-term memory deficits in the subpopulation of CAG140 mice that had a decreased ability to detect novel objects.

Ampakines are currently undergoing clinical trials for other central nervous system disorders, and seem to be well tolerated. This study provides a rationale for investigating whether such compounds might ameliorate the cognitive defects associated with $\mathrm{HD}$, for which there are currently no treatments.

Charlotte Harrison

ORIGINAL RESEARCH PAPER Simmons, D. S. et al. Up-regulating BDNF with an ampakine rescues synaptic plasticity and memory in Huntington's disease knockin mice. Proc. Natl Acad. Sci. USA 5 Mar 2009 (doi:10.1073/pnas. 0811228106) 